[0212-7199 (2005) 22: 1; pp 21-23] ANALES DE MEDICINA INTERNA Copyright (C) 2005 ARAN EDICIONES, S.L.

AN. MED. INTERNA (Madrid) Vol. 22, N. ${ }^{\circ} 1$, pp. 21-23, 200

\title{
Lamivudina en el tratamiento de la hepatopatía crónica por virus hepatitis B AgHbe minus
}

\author{
J. A. CARNEROS MARTÍN, C. DE LA COBA ORTIZ, E. MARTÍNEZ NÚÑEZ1, \\ P. FRADEJAS SALAZAR, A. ÁLVAREZ DELGADO, F. SÁNCHEZ MARTÍN, \\ M. RODRIGO RODRIGUEZ, F. GONZÁLEZ SAN MARTÍN
}

Servicio de Aparato Digestivo. Hospital Universitario. Salamanca. ${ }^{1}$ CAP Emigrantes II. Área 4 de Atención Primaria. Madrid

LAMIVUDINE FOR THE TEATMENT OF PATIENTS WITH HEPATITIS B AGHBE MINUS

\section{RESUMEN}

Introducción: Se estima que la hepatopatía crónica por virus de la hepatitis B afecta a más de 350 millones de personas en todo el mundo. Los pacientes AgHbe minus representan en algunas áreas entre el 50$80 \%$ del total. En estos pacientes la remisión espontánea es rara, la respuesta a interferón menor y la probabilidad de evolución a cirrosis y hepatocarcinoma mayor que en la cepa salvaje.

Objetivo: Analizar la respuesta al tratamiento con Lamivudina en pacientes con hepatopatía crónica VHB AgHBe negativos. Resultados: De los nueve pacientes tratados en nuestro servicio durante más de 3 meses, 7 eran AgHbe negativos. De ellos 6 pacientes respondieron al tratamiento, en un tiempo medio de 3,5 meses (rango 1-6). Se han producido dos recidivas a los 18 y 24 meses que han sido tratadas con Adefovir. Cuatro pacientes persisten con ADN negativo y transaminasas normales tras un tiempo medio de tratamiento de 25 meses.

Conclusiones: En nuestra serie, la mayoría de los pacientes $(77,7 \%)$ eran AgHbe negativos al inicio del tratamiento. La eficacia del tratamiento con Lamivudina en ellos es alta $(85,7 \%$ ) y precoz (media de 3,5 meses). En un tercio de los pacientes tratados se produce recidiva viral, al menos tras 1 año y medio de tratamiento.

PALABRAS CLAVE: Virus de la Hepatitis B. Antígeno e de la hepatitis B. Lamivudina.

\begin{abstract}
Introduction: It is estimated that chronic hepatitis B affects to than 350 million people around the world. Patients with eAgHB- minus account, in some areas, for between 50-80\% of the total of the population with chronic hepatitis B. Spontaneous clearance is rare within these patients, the response to interferon is low and the probability of developing cirrhosis and hepatocarcinoma is higher than in the wild type.

Aim: To analyze the response to lamivudine treatment in patients with chronic hepatitis $B$ which are $\mathrm{A} A \mathrm{AHB}$ negative.

Results: Seven of the 9 patients which were treated in our department for more than 3 months were eAgHB negative. Six of them responded to the treatment in an average time of 3.5 months (range $1-6$ months). There were two patients that relapsed at 18 and 24 months and they were treated with adefovir. Four patients remained DNA negative and had normal aminotransferases values after an average treatment time of 25 months.

Conclusion: In our series, the majority of the patients (77.7\%) were eAgHB negative at the beginning of treatment. The efficacy of the treat ment with lamivudine in these cases is high (85.7\%) and with an early response (average 3,5 months). One third of patients treated relapsed after one and a half years of treatment.
\end{abstract}

KEY WORDS: Hepatitis B virus. Antigen e. Lamivudine.

Carneros Martín JA, De la Coba Ortiz C, Martínez Núñez E, Fradejas Salazar P, Álvarez Delgado A, Rodrigo Rodríguez M, González San Martín F. Lamivudina en el tratamiento de la hepatopatía crónica por virus hepatitis B AgHbe minus. An Med Interna (Madrid) 2005; $22: 21-23$.

\section{INTRODUCCIÓN}

La hepatopatía crónica por el virus de la hepatitis B (VHB) afecta a más de 350 millones de personas en todo el mundo (1). El área de distribución del virus es mundial aunque existen zonas especialmente endémicas como Asia, África subsahariana, Australia, Nueva Zelanda y Sudamérica (2). En Estados Unidos se estima que hay 1,25 millones de portadores crónicos, definidos como positivos para el antígeno de superficie AgHbs durante más de 6 meses. España es un país con una incidencia intermedia y pese a que los casos de infecciones agudas están disminuyendo, debido a la mejora de las condiciones higiénico sanitarias y a la generalización de la vacunación, existe todavía un número importante de personas con infección crónica (3). De ellos entre el 15 y $40 \%$ desarrollarán complicaciones en relación con una hepatopatía crónica progresiva (cirrosis hepática y hepatocarcinoma) $(4,5)$.

La afectación hepática es más severa y rápidamente progresiva en pacientes con hepatopatía crónica VHB con el AgHbe negativo y el AcHbe positivo, que representan en algunas áreas el 50-80\% del total (6). Estos pacientes se caracterizan por la presencia de hepatopatía crónica VHB (con niveles de ADN viral detectables en suero y actividad necroinflamatoria en la biopsia hepática) pero con AgHbe 
negativo debido a la presencia de mutaciones en la región precore o core (7). La más frecuente, G1896A, crea un codón de parada prematura en la región precore que impide la producción de AgHbe (8). La tasas de respuesta sostenida en pacientes con hepatopatía crónica VHB con mutante precore tratados con interferón son menores que en los pacientes con la cepa salvaje (9) lo que obliga a la búsqueda de alternativas en el tratamiento de estos pacientes.

El objetivo de nuestro estudio es analizar la eficacia del tratamiento con Lamivudina en pacientes con hepatopatía crónica VHB AgHbe negativos (mutante precore).

\section{PACIENTES Y MÉTODOS}

En el Servicio de Aparato Digestivo del Hospital Universitario de Salamanca han sido tratados 12 pacientes con hepatopatía crónica por VHB con Lamivudina $100 \mathrm{mg} /$ día por vía oral. Nueve llevan más de 3 meses de tratamiento, y, de ellos, $7(77,7 \%)$ eran AgHbe negativo al inicio del tratamiento. Tres pacientes habían sido previamente tratados con interferón alfa 2 b durante un tiempo medio de 10 meses. En dos de ellos no había existido respuesta y el tercero presentó recidiva tras retirar el tratamiento.

Todos los pacientes fueron evaluados en consultas externas como mínimo una vez cada 3 meses con anamnesis y determinaciones de laboratorio que incluían, entre otras, transaminasas, AgHbs y DNA viral. Definimos la respuesta al tratamiento como la normalización de las transaminasas y la negativización del ADN viral (determinado en nuestro caso por técnicas de PCR). La recidiva implicaba la elevación de transaminasas acompañada de positivización de ADN de VHB.

\section{RESULTADOS}

Cuatro pacientes eran varones. La edad media al inicio del tratamiento era de 46,5 años (rango 31-64).

De los 7 pacientes con hepatopatía crónica VHB AgHbe negativo tratados con Lamivudina, $6(85,7 \%)$ han presentado respuesta al tratamiento. El tiempo medio de respuesta fue de 3,5 meses (rango 1-6).

Dos pacientes de los 6 con respuesta al tratamiento $(33,3 \%)$ han recidivado con aumento de transaminasas y ADN viral positivo a los 18 y 24 meses de iniciado el mismo.

En ambos se administró posteriormente Adefovir con respuesta en uno de ellos (en el otro paciente se suspendió el tratamiento en el primer mes por intolerancia digestiva).

Cuatro pacientes $(66,6 \%)$ persisten con ADN negativo y transaminasas normales tras un tiempo medio de 25 meses de tratamiento (rango 16-48). En ningún paciente se ha producido la negativización del AgHbs.

\section{DISCUSIÓN}

El diagnóstico de pacientes con hepatopatía crónica por VHB AgHbe negativo se basa en la presencia de AgHbs durante más de 6 meses, AgHbe indetectable con presencia de AcHbe, ADN del VHB, elevación de ALT y actividad necroinflamatoria en la biopsia hepática (10). La distribución de esta variante e minus es mayor de lo que se creía inicialmente, encontrándose casos en prácticamente todo el mundo y no únicamente en el área mediterránea y Asia como se había descrito (11). En nuestra serie representan el 77,7\% del total de pacientes con hepatopatía crónica VHB.

Los pacientes AgHbe negativos tienen habitualmente una edad mayor que los AgHbe positivos y existe un mayor predominio de varones (10). Además, en estos pacientes la enfermedad suele tener mayor actividad y ser más avanzada. De esta forma hasta el $38 \%$ de los pacientes tienen cirrosis en el momento del diagnóstico (12). Estos datos (edad mas avanzada y mayor grado de daño hepático) han hecho suponer que representa un estadio más avanzado en la historia natural de la hepatopatía por VHB (10).

La Lamivudina es un análogo de nucleósidos que se administra por vía oral y, tras su fosforilación, compite por la incorporación en la cadena de ADN del VHB provocando su terminación prematura (13) sin interferir con la síntesis de ADN humano. En pacientes con hepatopatía crónica por virus hepatitis B AgHbe negativos la Lamivudina consigue tasas de repuesta bioquímica y virológica del 70-96\% tras 48 semanas de tratamiento frente a $<10 \%$ en los pacientes en los que se administró un placebo $(14,15)$. Las tasas de mejoría histológica varían ampliamente entre el 20 y $95 \%$ (16).

Sin embargo, la mayoría de los pacientes $(90 \%)$ recidivan, con elevación de las transaminasas y reaparición del ADN viral, en cuanto se suspende el tratamiento $(16,17)$. La administración indefinida del fármaco tampoco es la solución debido a la aparición de mutaciones en el dominio YMDD de la ADN polimerasa viral que confieren resistencia al fármaco (18-20). De esta forma al final del primer año de tratamiento el $19 \%$ de los pacientes han desarrollado mutaciones, cifra que se eleva al $44 \%$ al final del segundo año (21). El mantenimiento del tratamiento con Lamivudina tras la aparición de mutaciones no ha demostrado ser de utilidad por lo que en la actualidad se recomienda su suspensión o sustitución (22).

La única alternativa actual al tratamiento con Interferón o Lamivudina es el Adefovir Dipivoxil. Este fármaco, que ha sido recientemente aprobado para el tratamiento de la hepatopatía crónica VHB, es un profármaco del Adefovir, un análogo sintético del deoxiadenosín-monofosfato. Presenta una potente actividad antiviral frente a la ADN polimerasa de hepadnavirus, retrovirus y herpesvirus y tiene las ventajas de su administración oral, la práctica ausencia de efectos adversos y la hasta ahora inusual aparición de mutaciones que condicionen la resistencia al fármaco $(23,24)$. Este último aspecto puede influir en que se convierta en la primera opción de tratamiento en pacientes con mutante precore, en los que la necesidad de tratamiento a largo plazo con Lamivudina puede favorecer la aparición de mutaciones.

En conclusión, en nuestra serie la mayoría de los pacientes con hepatopatía crónica VHB tienen la variante e minus $(77,7 \%)$, en ellos la eficacia del tratamiento con Lamivudina $100 \mathrm{mgs} /$ día por vía oral es alta $(85,7 \%)$, consiguiéndose de forma precoz (media de 3,5 meses) la normalización de las transaminasas y la negativización del ADN viral. En un tercio de los pacientes se produce recidiva viral al menos tras 1 año de tratamiento, en ellos el Adefovir es una alternativa de tratamiento. 


\section{Bibliografía}

1. Lee W. Hepatitis B virus infection. N Engl J Med 1997; 337: 17331745.

2. Lok ASF, McMahon BJ. Chronic hepatitis B. Hepatology 2001; 34: 1225-1241.

3. Sola R, Cruz De Castro E, Hombrados M, Planas R, Coll S, Jardi R, et al. Prevalence of hepatitis $\mathrm{B}$ and hepatitis $\mathrm{C}$ viruses in differente counties of Catalonia, Spain: cross-sectional study. Med Clin (Barc) 2002; 119: 90-95.

4. McMahon BJ. Hepatocellular carcinoma and viral hepatitis. In: Wilson RA, ed. Viral Hepatitis. New York: Marcel Dekker 1997; 315-330.

5. Maddrey WC. Hepatitis B: an importan public health issue. J Med Virol 2000; 61:362-366.

6. Huy TT, Ushijima H, Win KM, Luengrojanakul P, Shrestha PK, Zhong $\mathrm{ZH}$, et al. High prevalence of hepatitis B virus pre-s mutant in countries where it is endemic and its relationship with genotype and chronicity. $\mathrm{J}$ Clin Microbiol 2003; 41:5449-5455.

7. Hunt CM, McGill JM, Allen MI, Condreay LD. Clinical relevance of hepatitis B viral mutations. Hepatology 2000; 31: 1037-44.

8. Okamoto H, Tsuda F, Akahane Y, Sugai Y, Yoshiba M, Moriyama K, Tanaka T, et al. Hepatitis B virus with mutations in the core promoter for an e antigen-negative phenotype in carriers with antibody to e antigen. J Virol 1994; 68: 8102-8110.

9. Lampertico P, Del Ninno E, Manzin A, Donato MF, Rumi MG, Lunghi $\mathrm{G}$, Morabito A, et al. A randomized, controlled trial of a 24-month course of interferon alfa $2 \mathrm{~b}$ in patients with chronic hepatitis B who had hepatitis B virus DNA without hepatitis B e antigen in serum. Heptology 1997; 26: 1621-1625.

10. Fattovich G. Natural history and prognosis of hepatitis B. Semin Liver Dis 2003; 23: 47-58.

11. Funk ML; Rosenberg DM, Lok ASF. World-wide epidemiology of HbeAg-negative chronic hepatitis B and associated precore and core promoter variants. J Viral Hepatol 2002; 9: 52-61.

12. Zarski JP, Marcellin P, Cohard M, et al. Comparison of antiHBe-positive and Hbe-antigen-positive chronic hepatitis B in France. J Hepatol 1994; 20: 636-640.

13. Doong SL, Tsai CH, Schinazi RF, Liotta DC, Cheng YC. Inhibition of the replication of hepatitis B virus in vitro by 2',3'-dideoxy-3'-thiacytidine and related analogues. Proc Natl Acad Sci U S A 1991; 88: 8495-8499.

14. Rizzetto M. Efficacy of lamivudine in HbeAg-negative chronic hepatitis B. J Med Viral 2002; 66: 435-451.

15. Gaia S, Volpes R, Smedile A, Paganin S, Actis GC, Rizzetto M. Lamivudine in anti-HbeAg positive chronic hepatitis B: response after one year of treatment. J Hepatol 2000; 32 (Supl. 2): 112.

16. Tassopoulos NC, Volpes R, Pastore G, Heathcote J, Buti M, Goldin RD, et al. Efficacy of lamivudine in patients with hepatitis B e antigen negative/hepatitis B virus DNA-positive (precore mutant) chronic hepatitis B. Lamivudine Precore Mutant Study Group. Hepatology 1999; 29: 889-896.

17. Santantonio T, Mazzola M, Iacovazzi T, Miglietta A, Guastadisegni A Pastore G. Long-tterm follow-up of patients with anti-Hbe/HBV DNApositive chronic hepatitis $\mathrm{B}$ treated for 12 months with lamivudine. $\mathrm{J}$ Hepatol 2000; 32: 300-6.

18. Lai CL, Chien RN, Leung NWY, Chang TT, Guan R, Tai DI, et al. A one-year trial of lamivudine for chronic hepatitis B. N Engl J Med 1998; 339: 61-68.

19. Liaw YF, Leung NW, Chang TT, Guan R, Tai DI, Ng KY, et al. Effects of extended lamivudine therapy in asian patients with chronic hepatitis B. Gastroenterology 2000; 119: 172-180.

20. Dienstag JL, Schiff ER, Wright TL, Perrillo RP, Hann HW, Goodman $\mathrm{Z}$, et al. Lamivudine as initial treatment for chronic hepatitis B in the United States. N Engl J Med 1999; 341: 1256-1263.

21. Buti M, Cotrina M, Jardi R, de Castro EC, Rodríguez-Frías F, SánchezÁvila $\mathrm{F}$, et al. Two years of lamivudine therapy in anti-Hbe-positive patients with chronic hepatitis B. J Viral Hepatol 2001; 8: 270-5.

22. Liaw YF, Chien RN, Yeh CT. No benefit to continue lamivudine therapy after emergence of YMDD mutations. Antivir Ther 2004; 9: 257-262.

23. Marcellin P, Chang T-T, Lim SG, Tong MJ, Sievert W, Shiffman MI, et al. Adefovir Dipivoxil for the treatment of hepatitis B e antigen-positive chronic hepatitis B. N Engl J Med 2003; 348: 808-816.

24. Hadziyannis S, Tassopoulos N, Heathcote E, Chang TT, Kitis G, Rizzetto M, et al. Adefovir Dipivoxil for the treatment of hepatitis B e antigen-negative chronic hepatitis B. N Engl J Med 2003; 348:800-807. 